\title{
QUEEN'S
UNIVERSITY
BELFAST
}

\section{Observation of plasma density dependence of electromagnetic soliton excitation by an intense laser pulse}

Sarri, G., Kar, S., Romagnani, L., Bulanov, S. V., Cecchetti, C. A., Galimberti, M., Gizzi, L. A., Heathcote, R.,
Jung, R., Kourakis, I., Osterholz, J., Schiavi, A., Willi, O., \& Borghesi, M. (2011). Observation of plasma density
dependence of electromagnetic soliton excitation by an intense laser pulse. Physics of Plasmas, 18(8), 080704. [080704]. https://doi.org/10.1063/1.3625261

Published in:

Physics of Plasmas

Document Version:

Publisher's PDF, also known as Version of record

Queen's University Belfast - Research Portal:

Link to publication record in Queen's University Belfast Research Portal

Publisher rights

(C) 2013 AlP Publishing LLC

\section{General rights}

Copyright for the publications made accessible via the Queen's University Belfast Research Portal is retained by the author(s) and / or other copyright owners and it is a condition of accessing these publications that users recognise and abide by the legal requirements associated with these rights.

Take down policy

The Research Portal is Queen's institutional repository that provides access to Queen's research output. Every effort has been made to ensure that content in the Research Portal does not infringe any person's rights, or applicable UK laws. If you discover content in the Research Portal that you believe breaches copyright or violates any law, please contact openaccess@qub.ac.uk. 


\section{saisar \\ Plasmas}

\section{Observation of plasma density dependence of electromagnetic soliton excitation by an intense laser pulse}

G. Sarri, S. Kar, L. Romagnani, S. V. Bulanov, C. A. Cecchetti et al.

Citation: Phys. Plasmas 18, 080704 (2011); doi: 10.1063/1.3625261

View online: http://dx.doi.org/10.1063/1.3625261

View Table of Contents: http://pop.aip.org/resource/1/PHPAEN/v18/i8

Published by the American Institute of Physics.

\section{Related Articles}

Electron acoustic waves in a magnetized plasma with kappa distributed ions

Phys. Plasmas 19, 082314 (2012)

Dissipative electromagnetic solitary waves in collisional plasmas

Phys. Plasmas 19, 082306 (2012)

Existence domains of arbitrary amplitude nonlinear structures in two-electron temperature space plasmas. I.

Low-frequency ion-acoustic solitons

Phys. Plasmas 19, 072320 (2012)

Cylindrical ion-acoustic solitary waves in electronegative plasmas with superthermal electrons

Phys. Plasmas 19, 062110 (2012)

Study of envelope electron acoustic solitary waves under transverse perturbations having kappa distributed hot electrons

Phys. Plasmas 19, 062109 (2012)

\section{Additional information on Phys. Plasmas}

Journal Homepage: http://pop.aip.org/

Journal Information: http://pop.aip.org/about/about_the_journal

Top downloads: http://pop.aip.org/features/most_downloaded

Information for Authors: http://pop.aip.org/authors

\section{ADVERTISEMENT}

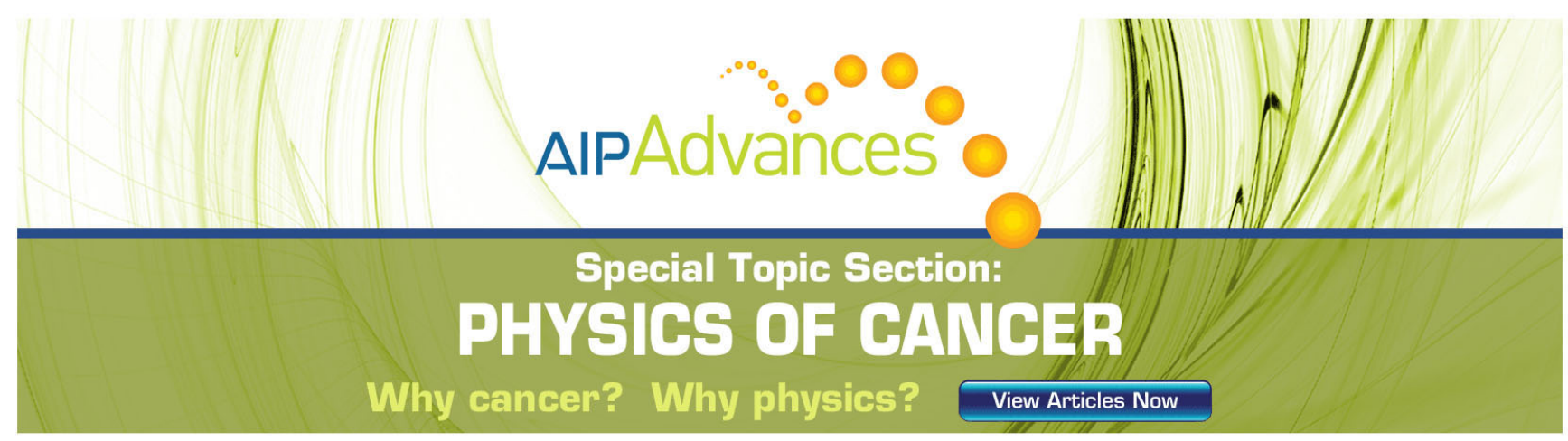




\title{
Observation of plasma density dependence of electromagnetic soliton excitation by an intense laser pulse
}

\author{
G. Sarri, ${ }^{1}$ S. Kar, ${ }^{1}$ L. Romagnani, ${ }^{2}$ S. V. Bulanov,${ }^{3}$ C. A. Cecchetti, ${ }^{4}$ M. Galimberti, ${ }^{5}$ \\ L. A. Gizzi, ${ }^{4}$ R. Heathcote,${ }^{5}$ R. Jung, ${ }^{6}$ I. Kourakis, ${ }^{1}$ J. Osterholz, ${ }^{6}$ A. Schiavi, ${ }^{7}$ \\ O. Willi, ${ }^{6}$ and M. Borghesi ${ }^{1}$ \\ ${ }^{1}$ Centre for Plasma Physics, The Queen's University of Belfast, Belfast BT7 1NN, United Kingdom \\ ${ }^{2}$ LULI, École Polytechnique, CNRS, route de Saclay, Palaiseau 91128, France \\ ${ }^{3}$ Kansai Photon Science Institute, JAEA, Kizugawa, Kyoto 619-0215, Japan \\ ${ }^{4}$ IPCF, Consiglio Nazionale delle Ricerche, CNR campus, Pisa, Italy \\ ${ }^{5}$ Rutherford Appleton Laboratory, Central Laser Facility, Chilton OX11 OQX, United Kingdom \\ ${ }^{6}$ Institute for Laser and Plasma Physics, Heinrich Heine University, Dusseldorf, Germany \\ ${ }^{7}$ Dipartimento di Energetica, Universitá di Roma 1 'La Sapienza', Roma, Italy
}

(Received 3 May 2011; accepted 26 July 2011; published online 10 August 2011)

\begin{abstract}
The experimental evidence of the correlation between the initial electron density of the plasma and electromagnetic soliton excitation at the wake of an intense $\left(10^{19} \mathrm{~W} / \mathrm{cm}^{2}\right)$ and short (1 ps) laser pulse is presented. The spatial distribution of the solitons, together with their late time evolution into post-solitons, is found to be dependent upon the background plasma parameters, in agreement with published analytical and numerical findings. The measured temporal evolution and electrostatic field distribution of the structures are consistent with their late time evolution and the occurrence of multiple merging of neighboring post-solitons. (C) 2011 American Institute of Physics. [doi:10.1063/1.3625261]
\end{abstract}

The propagation of a short, relativistically intense (dimensionless intensity $a_{0} \geq 1$ ), laser pulse through an underdense plasma is a physical scenario which attracts a great deal of attention in the scientific community mainly due to its possible applications which include laser wakefield acceleration $^{1}$ and fast ignition inertial confinement fusion. ${ }^{2}$ Such an interaction sets a very complex physical environment where both dispersion effects (mainly due to the finite inertia with which electrons respond to the laser electromagnetic field) and nonlinearities (arising from both the relativistic increase of the electron mass and the particle redistribution inside the plasma caused by ponderomotive forces) play significant roles in the plasma dynamics. ${ }^{3}$ Depending on the laser and plasma parameters, different non-linear coherent modes can in fact be excited in the wake of the laser pulse, providing one of the main mechanisms to transfer energy to electromagnetic (e.m.) fields in the plasma and to fast particles. During its propagation through a preformed plasma, the laser experiences, in fact, a significant energy loss which is mostly translated into a red shift of its wavelength. ${ }^{4}$ In sub-critical plasmas, this frequency decrease may be sufficient for the laser to locally experience an overcritical plasma, thus becoming trapped in plasma cavities. On an electron time scale, these cavities keep a constant shape with a characteristic radius of the order of the electron collisionless skin depth $\left(r_{e}=c / \omega_{p e}\right.$, where $\omega_{p e}$ is the Langmuir plasma frequency) and are usually referred to as e.m. solitons. ${ }^{5,7}$ The strong ponderomotive push, exerted by the trapped e.m. wave, expels the electron away from such plasma cavities leaving a positively charged core. On an ion time-scale, the Coulomb repulsion of the ions in the core causes these structures to radially expand and the soliton nature is lost: such late-time evolution of a soliton is thus commonly referred to as a post-soliton. ${ }^{8}$ Post-soliton expansion has been studied analytically, ${ }^{8,9}$ using the so-called snowplow model ${ }^{10}$ and the isolated spherical resonator model, ${ }^{11}$ and numerically, using particle-in-cell (PIC) codes. ${ }^{8}$ Numerical simulations show that their radial expansion induces neighboring post-solitons to merge in larger structures, with characteristic radii that exceed the typical dimensions of the isolated solitons. ${ }^{8,13}$ On the other hand, experimental observations of these phenomena are still limited; their first experimental observation was reported in Ref. 12, whereas the first temporally and spatially resolved characterization of isolated post-solitons can be found in Ref. 14 .

In this letter, we will present experimental evidence of the dependence of the e.m. soliton generation upon the initial electron density of the plasma. Copious formation of e.m. solitary structures occurs, at a distance of the order of the laser depletion length, only if the background electron density approaches a fraction of the non-relativistic critical density. The experimental findings suggest also that the plasma cavities detected are the result of multiple merging of neighboring post-solitons.

The experiment was carried out at the Rutherford Appleton Laboratory, employing the VULCAN Nd:glass laser system. ${ }^{15}$ 30J of linearly polarized $1 \mu \mathrm{m}$ laser light contained in $\tau_{L}=1 \mathrm{ps}$ full width half maximum pulse was focussed to a peak intensity of $10^{19} \mathrm{~W} \mathrm{~cm}^{-2}$ (dimensionless intensity $a_{0} \approx 2$ ) at the edge of a supersonic helium gas jet with a backing pressure ranging from 10 to 70 bar and an aperture of $2 \mathrm{~mm}$. Such a pulse was preceded by an amplified spontaneous emission (ASE) pedestal of 300 ps duration and a contrast ratio of $\approx 10^{-6}$. The interaction was mainly diagnosed via the proton radiography technique, ${ }^{16}$ which uses, as a particle probe, a laser accelerated proton beam, arising from the interaction of a secondary laser pulse $\left(\tau_{L} \approx 1 \mathrm{ps}, E_{L} \approx 30 \mathrm{~J}\right.$, and $I_{L} \approx 10^{19} \mathrm{~W} \mathrm{~cm}^{-2}$ ) with a 

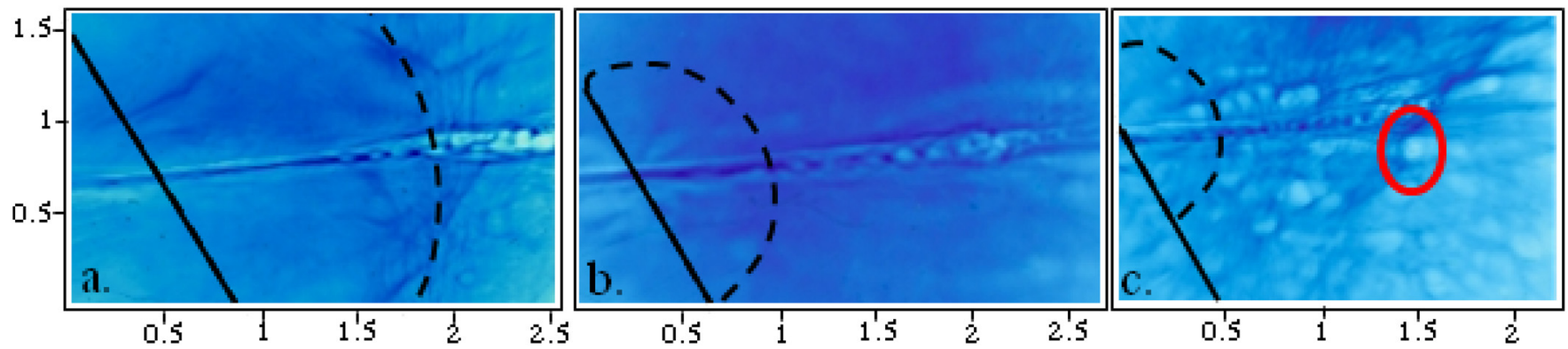

FIG. 1. (Color online) Proton radiographs of the laser plasma interaction, taken $20 \mathrm{ps}$ after the laser has reached its maximum intensity for an initial electron density of $0.23 n_{c}$ (a), $0.46 n_{c}$ (b), and $0.64 n_{c}$ (c). The laser is incident from the left side and the spatial scales are in mm. The solid lines represent the gas jet edge whereas the dashed lines represent circles of radius equal to the correspondent laser depletion length.

$10 \mu \mathrm{m}$ thick gold foil. The virtual point-like source ${ }^{17}$ allowed imaging of the interaction area with a geometrical magnification $M \approx(l+L) / l \approx 11$, where $l \approx 3 \mathrm{~mm}$ and $L \approx 3 \mathrm{~cm}$ (see Refs. 14 and 16 for a sketch of the experimental setup). The probe beam, after having passed through the gas jet, was recorded by a stack of RadioChromic Films. ${ }^{18}$ The interaction region was also diagnosed by Nomarsky interferometry, employing a frequency doubled laser pulse of low energy. The reconstructed electron density profiles along the propagation axis (not shown) showed that the ASE was able to fully ionize the gas inducing background electron densities ranging from 0.09 to 0.64 times the critical density for $1.05 \mu \mathrm{m}$ light $\left(n_{c} \approx 10^{21} \mathrm{~cm}^{-3}\right)$.

Typical proton radiographs of the interaction area are shown in Fig. 1 for different initial electron densities. All radiographs show a density-depleted channel, in correspondence to the laser propagation axis, which presents round plasma cavities within its walls. The channel formation mechanism and the appearance of such hybrid soliton-vortices structures within the channel walls are discussed in Refs. 19 and 20, respectively, and we refer the reader to those for a detailed description. Here, we focus our attention on the e.m. solitary structures appearing outside the channel, i.e., far off the main laser propagation axis. For an initial electron density of $n_{e}=0.23 n_{c}$ (Fig. 1(a)), a perturbed region is detected, starting at $\approx 1.3 \mathrm{~mm}$ from the gas jet edge, but no evidence of the occurrence of plasma cavities outside the channel can be found. Interactions at $n_{e}=0.09 n_{c}$ (not shown) evidenced a qualitatively similar behavior. At higher electron densities $\left(n_{e}=0.46 n_{c}\right.$, Fig. 1(b)), few plasma cavities start to appear outside the main channel and it is only when the electron density is increased to $0.64 n_{c}$ (Fig. 1(c)) that copious formation of cavities is detected. In this case, such structures appear throughout the entire extension of the plasma and, therefore, at large transverse distances. This can be understood by considering that the laser beam is likely to break up into several filaments approximately equally distributed over a wide angle, as both experimentally detected ${ }^{21}$ and theoretically predicted $^{22}$ for similar conditions. It is, therefore, reasonable to assume that a considerable fraction of the laser energy will be present also far from the main propagation axis.

These results can be interpreted by considering that the laser energy depletion will occur on a spatial scale of the order of $l_{\text {depl }} \approx l_{\text {pulse }}\left(\omega_{L} / \omega_{\text {pe }}\right)^{2}$ (Refs. 4 and 12) where $l_{\text {pulse }}$ represents the laser pulse length and $\omega_{L}\left(\omega_{p e}\right)$ represents the laser (plasma) frequency. This formula assumes that the laser energy is mostly dissipated through the excitation of plasma oscillations that are far from breaking and neglects high-frequency dissipation mechanisms such as ponderomotive effects and Raman scattering, a reasonable approximation for relatively long laser pulses $\left(\tau_{L} \omega_{p e} \geq 2 \sqrt{2}\left|a_{0}\right|^{-1}\right)$ and underdense plasmas. ${ }^{4}$ An initial plasma electron density of $0.09 n_{c}$ would imply a depletion length of about $3.8 \mathrm{~mm}$ (i.e., exceeding the extension of the plasma medium) whereas an initial electron density of $0.64 n_{c}$ reduces the depletion length down to $500 \mu \mathrm{m}$ (see Table I). Circles of radius equal to the correspondent depletion length are overplotted in Fig. 1: solitary structures start to appear only after a distance comparable to $l_{\text {depl }}$. Moreover, the ratio between the trapped and the initial laser energy $\left(k_{a b s}\right)$, which can be roughly approximated as $k_{a b s} \approx 1-\omega_{p e} / \omega_{L}$, should be of about $70 \%$ in the case of $n_{e}=0.09 n_{c}$ and $25 \%$ for $n_{e}=0.64 n_{c}$ (see Table I). It is thus clear that the efficient soliton creation occurs only when the laser depletion length is well within the plasma extension and the conversion efficiency is in the range 30\%-40\%. This result is consistent with published theoretical models of these structures (see, for instance, Ref. 5) which indicate that a maximum of $40 \%$ of the laser energy can be trapped in e.m. soliton structures.

Thanks to the multi-frame capability of the proton radiography technique, ${ }^{16}$ it has been possible to follow the temporal evolution of such structures in a temporal window ranging from 1 to $40 \mathrm{ps}$ after the peak of the laser pulse. The bubbles are seen already at 1-2 ps, i.e., when the laser is still propagating inside the gas jet. Once created, they appear to be stationary in the laboratory reference frame and to radially expand preserving a roughly circular shape. It must be noted that the soliton nature of these structures will in fact

TABLE I. Summary of the relevant plasma parameters for the different initial gas jet pressures discussed in the text. $l_{\text {exp }}$ represents the minimum distance from the gas jet edge at which post-solitons outside the laser axis have been detected.

\begin{tabular}{lcccccc}
\hline \hline$s \rho$ (bar) & $n_{e} / n_{c}$ & $\omega_{L} / \omega_{p}$ & $r_{e}(\mu \mathrm{m})$ & $l_{\text {depl }}(\mathrm{mm})$ & $l_{\text {exp }}(\mathrm{mm})$ & $k_{a b s}$ \\
\hline 10 & 0.09 & 3.55 & 0.56 & 3.8 & $/$ & 0.72 \\
25 & 0.23 & 2.22 & 0.35 & 1.5 & 1.3 & 0.55 \\
50 & 0.46 & 1.57 & 0.25 & 0.7 & 0.6 & 0.36 \\
70 & 0.64 & 1.33 & 0.21 & 0.5 & 0.4 & 0.25 \\
\hline \hline
\end{tabular}


persist only as long as the ion motion is not significant, i.e., for a time that is less than the inverse of the ion plasma frequency (of the order of 270 fs for $n_{e}=0.64 n_{c}$ ). This timescale lays below the resolution of the proton radiography technique (of the order of few picoseconds, see Ref. 16) explaining why the pure soliton stage could not be detected.

As an example, the temporal evolution of the diameter of the structure outlined by the red circle in Fig. 1(c) has been fitted using the expansion laws analytically derived by Bulanov and Pegoraro ${ }^{9}$ in the framework of the snowplow model. These analytical results predict the post-soliton expansion to be dependent upon the dimensionality of the expansion (i.e., spherical, cylindrical, or planar) and to occur with a typical time scale $\tau_{s}=\sqrt{2 \pi d_{0}^{2} n_{i} m_{i} /\left\langle E_{0}^{2}\right\rangle}$ where $d_{0}$ represents the initial soliton diameter $\left(d_{0}=2 r_{e}\right.$ in the case of an isolated soliton), $n_{i}\left(m_{i}\right)$ is the ion density (mass), and $\left\langle E_{0}^{2}\right\rangle$ is the time average of the square of the initial oscillating electric field inside the postsoliton. Assuming $k_{a b s} \approx 30 \%$ of the laser energy to be trapped in solitons (see Table 1), the trapped e.m. field in each cavity can be assumed to be of the order of $\left\langle E_{0}^{2}\right\rangle^{1 / 2}=\sqrt{k_{a b s}} \times\left\langle E_{L}^{2}\right\rangle^{1 / 2} \approx 3 \times 10^{12} \mathrm{~V} / \mathrm{m}$. Under this assumption, $\tau_{s}=0.1 d_{0}$ with $\tau_{s}$ and $d_{0}$ expressed in picoseconds and microns, respectively. For our experimental conditions and assuming only the initial diameter of the structure $d_{0}$ as free parameter, the analytical scalings for spherical and cylindrical expansion reported in Ref. 9 will read, respectively

$$
d(t)=d_{0}\left[\frac{5 t}{0.1 d_{0}}\right]^{2 / 5}, \quad d(t)=d_{0}\left[\frac{3^{3 / 2} t}{0.1 d_{0}}\right]^{1 / 3} .
$$

The expansion data are fitted in Fig. 2 using these two formulas; a cylindrical scaling gives a better fit, as expected for postsolitons created at the wake of a linearly polarized laser pulse. ${ }^{14}$ However, both the cylindrical and spherical scalings predict an initial diameter of the structure of about $d_{0} \approx 15-20 \mu \mathrm{m}$ which greatly exceeds the one expected for an isolated post-soliton $\left(2 r_{e} \approx 0.4 \mu \mathrm{m}\right)$. This can be explained by taking into account that, already at the very early stage of its lifetime, the post-soliton has experienced multiple merging with its neighbors. Assuming a uniform soliton distribution inside the volume

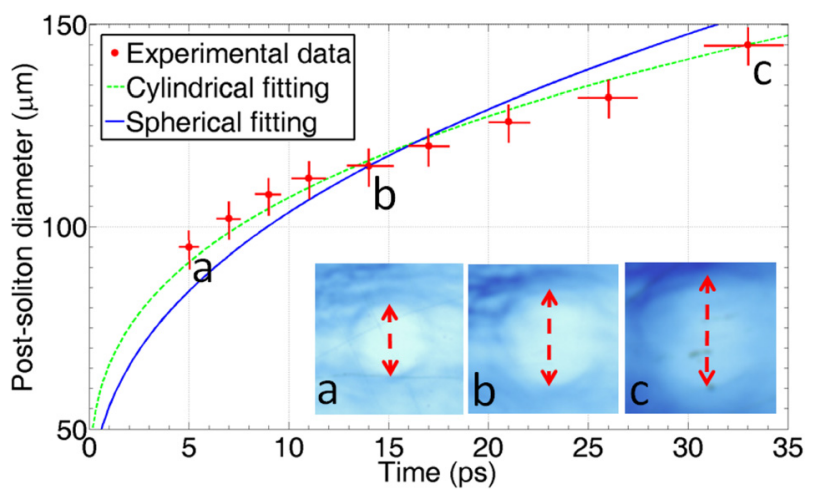

FIG. 2. (Color online) Diameter of the bubble outlined by the red circle in Fig. 1(c) as a function of time and fits using the results from the snowplow model for cylindrically and spherically symmetric expansions. The insets depict zooms of the post-soliton at different times. Other bubbles in Fig. 1(c) have been found to behave in a similar manner. traced by the laser pulse (as corroborated by the experimental data, see Fig. 1(c)), the average inter-soliton distance will be of the order of $D_{s} \approx r_{e} /\left[k_{a b s}\left(l_{\text {pulse }} / l_{\text {depl }}\right)\right] \mathrm{s}^{1 / 3} \approx 0.4 \mu \mathrm{m},{ }^{12}$ which is comparable to the soliton radius. Once the post-solitons start to radially expand, they will thus immediately tend to merge with a characteristic time $t_{\text {merg }}=\tau_{s}\left[l_{\text {depl }} /\left(k_{\text {abs }} l_{\text {pulse }}\right)\right]^{1 / 3} \approx 1-2 \mathrm{ps}$, which is only few times the ion plasma period. The structures seen in the proton radiographs will thus be already the result of multiple post-soliton merging and will, therefore, have an initial diameter that is much larger than the one expected from a single structure.

The electrostatic field distribution associated with these structures also indicates that their observed evolution is at a late stage. It is well known that e.m. solitons in plasmas will be associated with two completely distinct components of the electric field: a purely e.m. one, associated with the trapped radiation and oscillating at a frequency of the order of a fraction of the initial laser e.m. field and a purely electrostatic one, associated with the charge separation induced within the plasma. The first component is oscillating too fast to be detected by the probing protons which will thus average it down to zero, whereas the second one, which is quasistatic, will be responsible for the proton deflections. It is worth mentioning that no significant quasi-static magnetic fields should be present around the post-soliton (see, for instance, the simulation results reported in Ref. 14) and can thus be neglected in analyzing the probing protons deflections. A lineout of the probing proton density distribution in correspondence to the post-soliton outlined in Fig. 1(c) is plotted in Fig. 3(d) (see Fig. 3(a) for the crosscut direction). This figure shows that the soliton walls are much wider than the one expected by the snowplow model (compare results in Ref. 14). This is consistent with the late time stage of these structures. The electrostatic potential $\left(\phi_{s}\right)$ induced by the plasma charge separation associated with the post-soliton, is in fact expected to have different profiles depending on the ratio of its amplitude to the vector potential of the trapped e.m. wave $\left(A_{s}\right)$. If $\phi_{s} \gg A_{s}$, the potential will be peaked at the soliton walls, consistently with the snowplow model. Such a strong electrostatic potential is possible only at the preliminary stage of the soliton life, time at which an almost
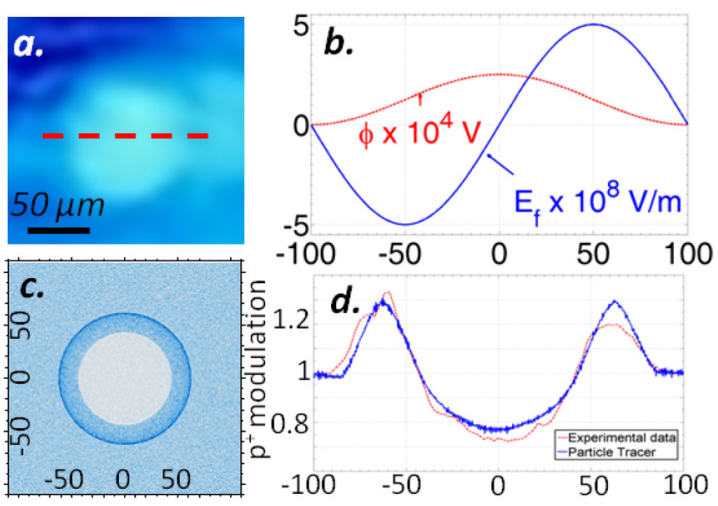

FIG. 3. (Color online) (a) Zoom of the bubble outlined by the red circle in Fig. 1(c). (b) Electric field used as an input for the particle tracing code and related potential. (c) Simulated proton deflection. (d) Comparison between the simulated and experimental proton deflection along the red dashed line in the frame (a). All the spatial scales are in microns. 
complete electron depletion is expected in the core of the soliton. On the other hand, for $\phi_{s} \ll A_{s}$, the potential will rather show a positive mono-polar distribution centered with the soliton. ${ }^{23}$ Such a potential is related to a bipolar electrostatic field and to much wider soliton walls compared to the snowplow model, as the lineout in Fig. 3(d) suggests. A bipolar electrostatic field, spherically symmetric around its center, has thus been used as input for particle tracer simulations. ${ }^{24}$ This code traces proton trajectories from a pointlike source through a given three-dimensional electric field distribution giving, as a result, a two-dimensional proton density map at the detector plane. The finite spatial extension of the proton source (of the order of 5-10 $\mu \mathrm{m}$, see Ref. 17) is taken into account by a subsequent convolution of the particle tracer results. The experimental probing proton distribution is well reproduced by the simulated results (Fig. 3(c)) if an electrostatic field as the one in Fig. 3(b) and a proton source extension of $8 \mu \mathrm{m}$ are considered (see Fig. 3(d) for the comparison). This field is associated with a positive potential of maximum amplitude of $2.5 \times 10^{4} \mathrm{~V}$ (consistent with the a priori assumption $\phi_{s} \ll A_{s}$ ). Similar electric field shapes reproduce the experimental data also at later times, providing that a larger width and a smaller amplitude is considered.

In conclusion, experimental evidence of copious formation of electromagnetic post-soliton structures following the interaction of a relativistically intense laser pulse with a underdense helium plasma has been presented. The experimental data evidence a clear dependence of soliton excitation upon the initial plasma density, in line with published theoretical findings. The electric field distribution of such structures suggests the late-time evolution of these structures whereas their expansion suggests that they might be the result of multiple coalescence of neighboring post-solitons.

We acknowledge the support of the RAL/CLF staff. This work has been supported by the Engineering and Physical Sciences Research Council [Grant Numbers EP/E035728/1 (LIBRA consortium) and EP/D06337X/1], by British Council-MURST-CRUI, and by DFG TR 18 and GK 1203.

${ }^{1}$ W. P. Leemans, B. Nagler, A. J. Gonsalves, Cs. Tóth, K. Nakamura, C. G. R. Geddes, E. Esarey, C. B. Schroeder, and S. M. Hooker, Nature Phys. 2, 696 (2006); V. Malka, S. Fritzler, and E. Lefebvre, Science 298, 1596 (2002).

${ }^{2}$ M. Tabak, J. Hammer, M. E. Glinsky, W. L. Kruer, S. C. Wilks, J. Woodworth, E. M. Campbell, and M. D. Perry, Phys. Plasmas 1, 1626 (1994).
${ }^{3}$ W. Kruer, The Physics of Laser Plasma Interactions (Westview, Cambridge, MA, 2003).

${ }^{4}$ S. V. Bulanov, I. N. Inovenkov, V. I. Kirsanov, N. M. Naumova, and A. S. Sakharov, Phys. Fluids B 4, 1935 (1992).

${ }^{5}$ G. Lehmann, E. W. Laedke, and K. H. Spatschek, Phys. Plasmas 13, 092302 (2006).

${ }^{6}$ S. V. Bulanov, T. Zh. Esirkepov, N. M. Naumova, F. Pegoraro, and V. A. Vshivkov, Phys. Rev. Lett. 82, 3440 (1999).

${ }^{7}$ T. Esirkepov, K. Nishihara, S. V. Bulanov, and F. Pegoraro, Phys. Rev. Lett. 89, 275002 (2002).

${ }^{8}$ N. M. Naumova, S. V. Bulanov, T. Zh. Esirkepov, D. Farina, K. Nishihara, F. Pegoraro, H. Ruhl, and A. S. Sakharov, Phys. Rev. Lett. 87, 185004 (2001).

${ }^{9}$ S. V. Bulanov and F. Pegoraro, Phys. Rev. E 65, 066405 (2002).

${ }^{10}$ M. A. Leontovich and S. M. Osovets, At. Energ. 3, 81 (1956); Ya. B. Zel'dovich, and Yu. P. Raizer, Physics of Shock Waves and HighTemperature Hydrodynamic Phenomena (Academic, New York, 1967).

${ }^{11}$ L. D. Landau and L. M. Lifshits, Electrodynamics of Continuous Media (Pergamon, Oxford, 1984).

${ }^{12}$ M. Borghesi, S. Bulanov, D. H. Campbell, R. J. Clarke, T. Zh. Esirkepov, M. Galimberti, L. A. Gizzi, A. J. MacKinnon, N. M. Naumova, F. Pegoraro, H. Ruhl, A. Schiavi, and O. Willi, Phys. Rev. Lett. 88, 135002 (2002).

${ }^{13}$ S. V. Bulanov, F. Califano, T. Zh. Esirkepov, K. Mima, N. M. Naumova, K. Nishihara, F. Pegoraro, Y. Sentoku, and V. A. Vshivkov, Physica D 152, 682 (2001).

${ }^{14}$ G. Sarri, D. K. Singh, J. R. Davies, F. Fiuza, K. L. Lancaster, E. L. Clark, S. Hassan, J. Jiang, N. Kageiwa, N. Lopes, A. Rehman, C. Russo, R. H. H. Scott, T. Tanimoto, Z. Najmudin, K. A. Tanaka, M. Tatarakis, M. Borghesi, and P. A. Norreys, Phys. Rev. Lett. 105, 175007 (2010).

${ }^{15}$ C. Danson, J. Collier, D. Neely, L. J. Barzanti, A. Damerell, C. B. Edwards, M. H. R. Hutchinson, M. H. Key, P. A. Norreys, D. A. Pepler, I. N. Ross, P. F. Taday, W. T. Toner, M. Trentelman, F. N. Walsh, T. B. Winstone, and R. W. W. Wyatt, J. Mod. Opt. 45, 1653 (1998).

${ }^{16}$ G. Sarri, C. A. Cecchetti, L. Romagnani, C. M. Brown, D. J. Hoarty, S. James, J. Morton, M. E. Dieckmann, R. Jung, O. Willi, S. V. Bulanov, F. Pegoraro, and M. Borghesi, New J. Phys. 12, 045006 (2010).

${ }^{17}$ M. Borghesi, A. J. Mackinnon, D. H. Campbell, D. G. Hicks, S. Kar, P. K. Patel, D. Price, L. Romagnani, A. Schiavi, and O. Willi, Phys. Rev. Lett. 92, 05503 (2004).

${ }^{18}$ J. F. Dempsey, D. A. Low, S. Mutic, J. Markman, A. S. Kirov, G. H. Nussbaum, and J. F. Williamson, Med. Phys. 27, 2462 (2000).

${ }^{19}$ S. Kar, M. Borghesi, C. A. Cecchetti, L. Romagnani, F. Ceccherini, T. V. Liseykina, A. Macchi, R. Jung, J. Osterholz, O. Willi, L. A. Gizzi, A. Schiavi, M. Galimberti, and R. Heathcote, New J. Phys. 9, 402 (2007).

${ }^{20}$ L. Romagnani, A. Bigongiari, S. Kar, S. V. Bulanov, C. A. Cecchetti, T. Zh. Esirkepov, M. Galimberti, R. Jung, T. V. Liseykina, A. Macchi, J. Osterholz, F. Pegoraro, O. Willi, and M. Borghesi, Phys. Rev. Lett. 105, 175002 (2010).

${ }^{21}$ Z. Najmudin, K. Krushelnick, M. Tatarakis, E. L. Clark, C. N. Danson, V. Malka, D. Neely, M. I. K. Santala, and A. E. Dangor, Phys. Plasmas 10, 438 (2003).

${ }^{22}$ M. Grech, G. Riazuelo, D. Pesme, S. Weber, and V. T. Tikhonchuk, Phys. Rev. Lett. 102, 155001 (2009).

${ }^{23}$ V. Saxena, A. Das, S. Sengupta, P. Kaw, and A. Sen, Phys. Plasmas 14, 072307 (2007).

${ }^{24}$ A. Schiavi, Ph.D. thesis, Imperial College of Science, Technology and Medicine, London, UK, 2003. 